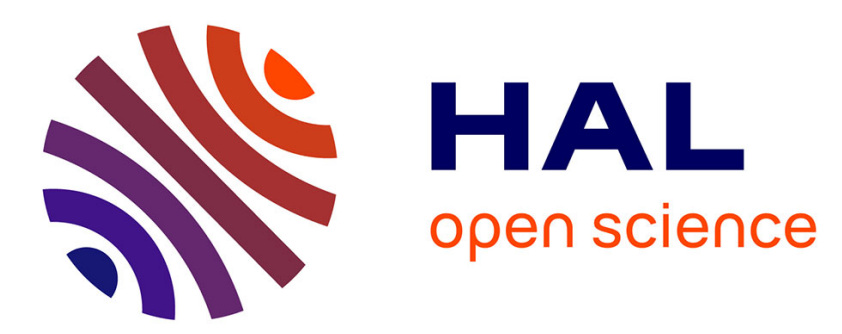

\title{
Ensemble électronique de comptage automatique de grains dans les émulsions nucléaires
}

\author{
A. Bernheim, M. Combes, J.C. Fayolle, H. de Surville, M. Morand
}

\section{To cite this version:}

A. Bernheim, M. Combes, J.C. Fayolle, H. de Surville, M. Morand. Ensemble électronique de comptage automatique de grains dans les émulsions nucléaires. Revue de Physique Appliquée, 1968, 3 (1), pp.5960. 10.1051/rphysap:019680030105900 . jpa-00242821

\section{HAL Id: jpa-00242821 https://hal.science/jpa-00242821}

Submitted on 1 Jan 1968

HAL is a multi-disciplinary open access archive for the deposit and dissemination of scientific research documents, whether they are published or not. The documents may come from teaching and research institutions in France or abroad, or from public or private research centers.
L'archive ouverte pluridisciplinaire HAL, est destinée au dépôt et à la diffusion de documents scientifiques de niveau recherche, publiés ou non, émanant des établissements d'enseignement et de recherche français ou étrangers, des laboratoires publics ou privés. 


\title{
ENSEMBLE ÉLEGTRONIQUE DE GOMPTAGE AUTOMATIQUE DE GRAINS DANS LES ÉMULSIONS NUGLÉAIRES
}

\author{
Par A. BERnheim, M. COMBES, J. G. FAYOLlE, H. De SURVILlE et M. MORAND, \\ Laboratoire de Physique Générale, Faculté des Sciences de Paris.
}

(Reçu le 6 juillet 196\%.)

\begin{abstract}
Résumé. - Un ensemble électronique réalisé à partir d'une chaîne de télévision en circuit fermé effectue le comptage automatique des grains d'une trace dans une émulsion nucléaire.

Abstract. - A device is described which, starting from a closed circuit television chain, provides automatic blob counting of tracks in nuclear emulsions.
\end{abstract}

I. But de l'étude. - Dans le cadre du problème du dépouillement automatique des émulsions nucléaires par l'intermédiaire d'un ensemble électronique de suivage de traces à partir d'une chaîne de télévision en circuit fermé [1], [2], [3], nous nous sommes proposé l'étude et la réalisation d'un dispositif automatique de comptage des grains.

II. Principe. - La détection est basée sur l'analyse grain par grain de la trace à l'aide d'un balayage. Le système analyseur d'image traduit par l'intermédiaire d'un signal électronique la présence d'un grain dans le champ d'observation.

Le principe est de compter ces signaux électroniques à l'aide d'un compteur à tubes afficheurs (Nixies) [4]. Deux problèmes se posent :

- L'analyse T.V. étant itérative, chaque grain est enregistré 50 fois par seconde par le compteur;

- Un grain occupant, dans notre cas, plusieurs lignes T.V., l'analyse fournit un signal à chaque ligne et un même grain est compté plusieurs fois.

1. Solution du premier problème. - On utilise des bascules bistables commutées par les signaux de synchronisation trame commandant l'ouverture d'une porte pendant l'analyse d'une demi-image (soit 1/50 de seconde; les grains occupant tous au moins deux lignes, nous sommes assurés de n'en sauter aucun).

Le diagramme du montage est représenté figure 1.

Le fonctionnement est le suivant :

État initial : La bascule B 1 est dans l'état voulu pour que la porte «ET » $\mathrm{n}^{0} 1$ soit fermée;

La bascule B 2 est dans l'état voulu pour que la porte «ET » no 2 soit fermée.

Les signaux « trames » et " grains » étant appliqués aux points précisés sur le diagramme, l'opération de

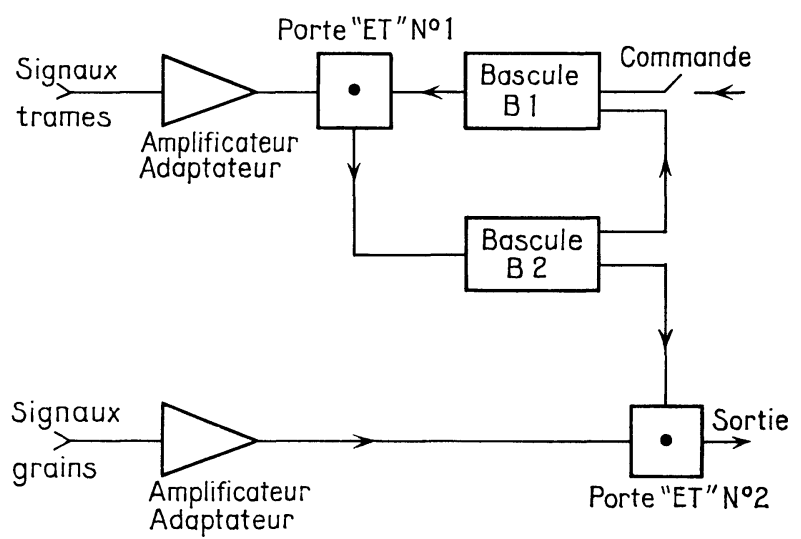

FIG. 1. - Diagramme du $1^{\mathrm{er}}$ montage.

comptage est déclenchée par l'opérateur. Celui-ci, à l'aide d'une commande manuelle, provoque le changement d'état de B 1; la porte «ET » 1 s'ouvre et les signaux trames sont alors appliqués à B 2 qui change d'état avec la première impulsion, déclenchant l'ouverture de la porte «ET » 2.

(L'entrée de $\mathrm{B} 1$ reliée à une sortie de $\mathrm{B} 2$ a été choisie de telle sorte que le premier changement d'état de B 2 soit sans effet sur B 1.)

La deuxième impulsion trame remet l'ensemble dans l'état initial (en effet, B 1 est cette fois-ci commutée par ce deuxième changement d'état de B 2).

Les signaux grains ont pu être récupérés à la sortie pendant que la porte «ET » 2 était ouverte, c'est-àdire durant l'intervalle de temps séparant la première impulsion trame de la seconde. L'analyse n'a eu lieu qu'une fois.

2. Solution du Deuxième PROBlème. - Un grain occupant $n$ lignes est représenté au niveau de l'analyse T.V. par $n$ impulsions espacées de $64 \mu$ s (64 $\mu$ s est 
le temps de balayage d'une ligne pour le standard 625). Un étalon de temps de $64 \mu$ s semblait donc nécessaire pour discriminer et éliminer, dans les $n$ impulsions représentatives d'un même grain, les $(n-1)$ impulsions ne devant pas parvenir au compteur. Nous avons utilisé une ligne à retard C.S.F. type Secam. Notre fréquence de travail étant 15625 périodes $\left(1 / 64 \times 10^{6}\right)$ et ce type de ligne étant prévu pour fonctionner aux alentours de $5 \mathrm{MHz}$, nous avons dû prévoir à l'entrée de la ligne la modulation d'un signal à $5 \mathrm{MHz}$ par les impulsions grains, impulsions récupérées à la sortie par un étage démodulateur.

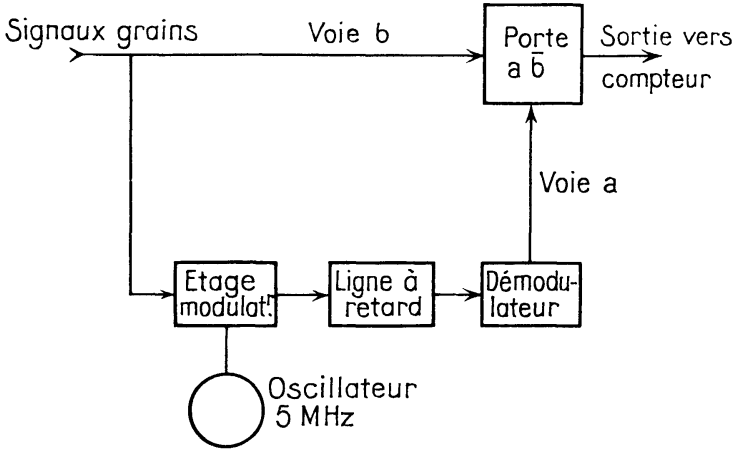

FIG. 2. - Diagramme du $2^{\mathrm{e}}$ montage.

Le diagramme du montage est représenté figure 2 . Le fonctionnement est le suivant :

La porte $a \bar{b}$ ne permet le passage d'une impulsion en provenance de la voie a qu'en l'absence d'impulsions en provenance de la voie $b$. C'est donc la dernière impulsion de la séquence qui est envoyée au compteur.

III. Réalisation. - 1. Premier montage. - Nous avons utilisé des portes à diodes. Leurs liaisons avec les bascules ont demandé la mise en place d'étages adaptateurs d'impédance non représentés sur le diagramme. Par ailleurs, la finesse des impulsions a nécessité l'emploi de transistors H.F. (bande passante minimum $2 \mathrm{MHz}$ ).

2. Deuxième montage. - Comme précédemment, de nombreux étages adaptateurs d'impédance ont été nécessaires en différents points, notamment aux extrémités de la ligne à retard (entrée et sortie sur $150 \Omega$ ). En outre, celle-ci introduit un affaiblissement consi-

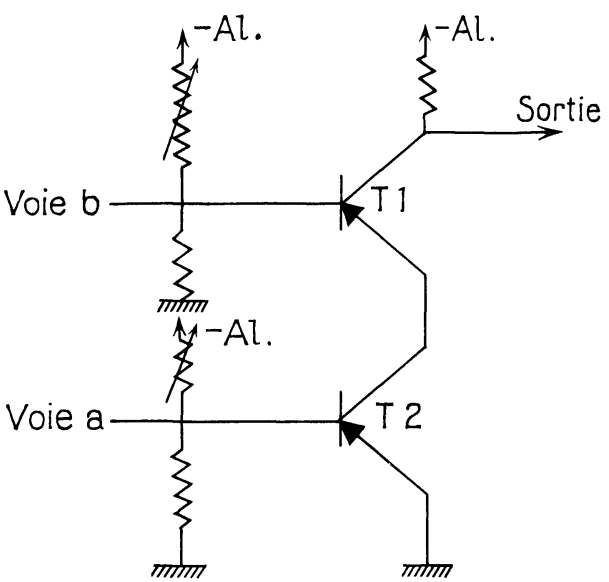

Frg. 3. - Schéma de la porte $a \bar{b}$.

dérable $(\leqslant 20 \mathrm{db})$, des étages amplificateurs ont été prévus avant la porte $a \bar{b}$. L'oscillateur est du type Colpitts. La porte $\mathrm{a} \overline{\mathrm{b}}$ utilise deux transistors : l'un normalement passant est bloqué par les impulsions en provenance de la voie b; l'autre normalement bloqué est rendu passant par les impulsions en provenance de la voie a. Ainsi seule la dernière impulsion en provenance de cette voie peut être récupérée sur le collecteur du premier transistor (fig. 3).

IV. Conclusion. - Montés en série avec les adaptateurs convenables, ces deux montages nous fournissent une seule impulsion par grain et permettent un comptage direct.

Nous avons donc réalisé un ensemble électronique permettant un comptage automatique de grains le long d'une trace de particule dans une émulsion nucléaire [5], [6].

Compte tenu de leur fréquence, les impulsions devront être enregistrées sur bande magnétique en vue de leur exploitation ultérieure par ordinateur.

Dans le cadre de l'ensemble électronique de suivage de traces, il sera possible d'étudier la distribution des paquets de grains («blobs ») ou des lacunes (《gaps »). En effet, on peut associer au comptage une mesure de longueurs en shuntant le deuxième montage; le diamètre d'un grain est alors proportionnel au nombre d'impulsions fournies.

Manuscrit reçu le 6 juillet 1967.

\section{BIBLIOGRAPHIE}

[1] BeRnheim (A.) et al., Nucl. Inst. and Meth., 1965, 34, 345 .

[2] Barbier (J. P.) et al., Communication VI Int. Conf. on Corpus Phot., Florence, 1966.
[3] BARBIER (J. P.) et al., Nucl. Inst. and Meth., 1967, 49, 38.

[4] Ul,RICH (J. P.), D.E.S., Paris, 1966.

[5] Combes (M.), D.E.S., Paris, 1967.

[6] DE Surville (H.), D.E.S., Paris, 1967. 